\title{
Design of Visual Servo Robot Tracking System Based on Image Feature
}

\author{
Yue Guo ${ }^{1, a}$, Xiaolan Yao ${ }^{2, b}$, Wei Li ${ }^{3, \mathrm{c}}$ \\ ${ }^{1,2,3}$ School of Automation, Beijing Institute of Technology, Beijing, 100081, China \\ aysugygy@163.com, byaoxiaolan@bit.edu.cn, c1439675880@qq.com
}

Keywords: Visual servo robot, Track a target, System development.

Abstract. The purpose of the paper is to design a visual servo robot system to finish some tasks with a better effect instead of workers. This system is designed with closed-loop control based on image feature, and it is more accurate than the control based on position. The system consists of two parts, including a control software system and a set of hardware system. The two parts are connected through a vision module, which collects the coordinate information of the target. In order to achieve good tracking precision, kinematics model and analysis of the experimental platform are given, and inverse kinematics is concluded accuracy. Finally, the control software is presented. With the experimental verification, the visual servo robot system can reach the goal of tracking the target with good performance of real-time and tracking precision.

\section{Introduction}

In recent years, robotic visual servo has become one of the most representative technology in the frontier of science and technology, and it greatly improves the industrial production capacity, profoundly influences the development of human society.

Piepmeier, McMurray and Lipkin proposed quasi Newton method to realize the fixed image tracking in the case of an uncalibrated camer [1]. Elbanhawi, Simic and Jazar presented a new algorithm to control the distance from waypoints to obstacles, and then the robot could avoid obstacles through the waypoints [2]. Silveira and Malis used the method of visual servo to track a moving targrt with good precision and robustness, and this method did not need any measurement information of the targe [3]. Scheggi, Morbidi and Prattichizzo put forward a multi-robot interaction mechanism, which used the camera measurement of the distance between each other and do coordinated motion [4].

In the paper, a visual servo robot system is designed to achieve the function of tracking a moving targrt precisely based on image feature. This paper is organized as follows. In section 2, composition and design of the system are given. In section 3, kinematics model and analysis of the experimental platform are presented. Working principle and process of the visual module is introduced in section 4 . And in section 5, software programming is completed. Experimental results and analysis in this paper are presented in section 6. Finally, work of this paper is summarized in the last section.

\section{Overall Design of the System}

The visual servo robot tracking system is designed with classical closed-loop control, including controller, actuator, controlled object and detector [5], as shown in Fig. 1.

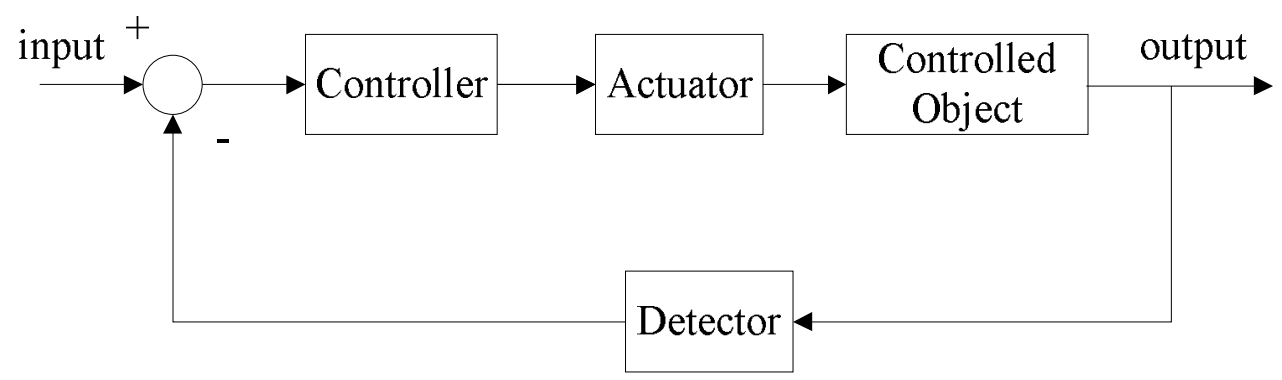

Fig. 1: Block diagram of the system 
The controller is made of two parts including control software and a motion control card(PMAC). The control software made by VS2010 and OpenCV sends control commands to the motion control card, and then the motion control card generates motion instructions to servo drivers.

The actuator consists of servo drivers, AC servo motors and reducers. Its function is to execute instructions from the controller.

The controlled object is a robot platform as shown in Fig. 2. It is suitable for the occasion in which the target moves with small trajectory and span. In addition, it has characteristics of high stability and high rigidity, so there is no dithering on the occasion of high-speed movement.

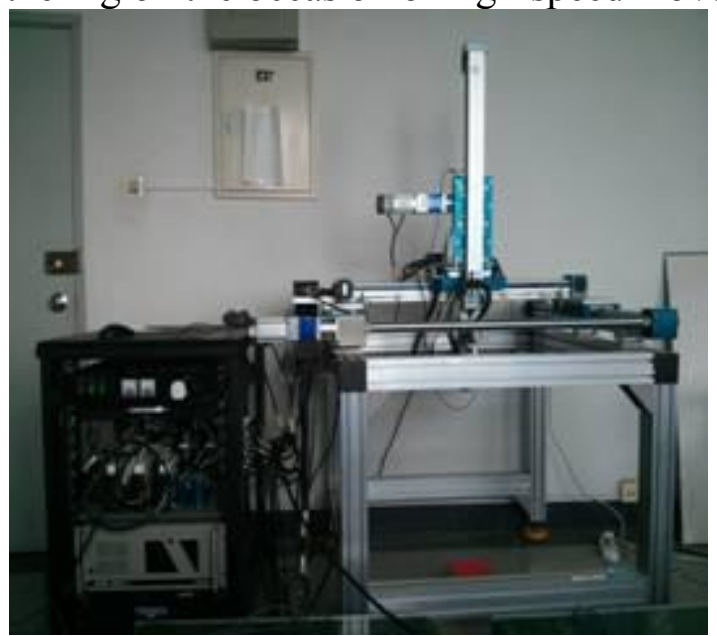

Fig. 2: Mechanical structure of the system

Dimension of mechanical structure: $1150 * 1050 * 1000[\mathrm{~mm}]$.

Actual motion range: $700 * 485 * 700[\mathrm{~mm}]$.

The detector plays an important role in the tracking system. It includes a camera, an image capture card and PC. The camera is CCD pinhole camera, whose vision model is shown in Fig. 3.

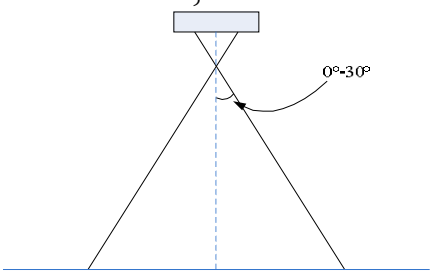

Fig. 3: Visual range of the camera

Installation height of the camera: $705[\mathrm{~mm}]$.

Angle range of the camera: $0^{\circ}-30^{\circ}$.

Video resolution of the image capture card: $720 * 576$.

\section{Kinematics Analysis of the Platform}

The world coordinate system is established on the base of the platform, and it is represented by $F_{W}\left(O_{W}-X_{W} Y_{W} Z_{W}\right)$. Then a camera coordinate system is established on the end of the camera, that is $F_{C}\left(O_{C}-X_{C} Y_{C} Z_{C}\right)$. The kinematics model of the platform is built in Fig. 4. 


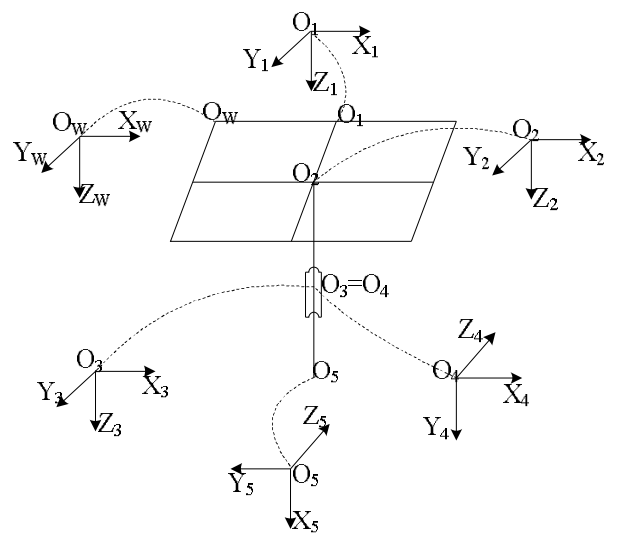

Fig. 4: Coordinate system of visual platform

So a target point in the world system and in the camera system can be separately represent by $P_{W}$ and $P_{C}$. Because the camera is installed at the end of the manipulator, so the relationship of them is shown in Eq. 1.

$P_{C}=T_{W} P_{W}$.

$T_{W}=\left({ }_{0}^{1} R_{1}^{2} R_{2}^{3} R_{3}^{4} R_{4}^{5} R\right)$.

$T_{W}$--Homogeneous transformation matrix from the world coordinate system to the camera coordinate system.

${ }_{i}^{i+1} R$--Homogeneous transformation matrix from $\operatorname{system} i$ to $\operatorname{system}(i+1)$.

${ }_{0}^{1} R=\left[\begin{array}{llll}1 & 0 & 0 & x \\ 0 & 1 & 0 & 0 \\ 0 & 0 & 1 & 0 \\ 0 & 0 & 0 & 1\end{array}\right]$.

${ }_{1}^{2} R=\left[\begin{array}{llll}1 & 0 & 0 & 0 \\ 0 & 1 & 0 & y \\ 0 & 0 & 1 & 0 \\ 0 & 0 & 0 & 1\end{array}\right]$.

${ }_{2}^{3} R=\left[\begin{array}{cccc}1 & 0 & 0 & 0 \\ 0 & 1 & 0 & 0 \\ 0 & 0 & 1 & z \\ 0 & 0 & 0 & 1\end{array}\right]$.

${ }_{3}^{4} R=\left[\begin{array}{cccc}\cos \alpha & 0 & \sin \alpha & 0 \\ \sin \alpha & 0 & -\cos \alpha & 0 \\ 0 & 1 & 0 & 0 \\ 0 & 0 & 0 & 1\end{array}\right]$.

${ }_{4}^{5} R=\left[\begin{array}{cccc}\cos \beta & -\sin \beta & 0 & a \cos \beta \\ \sin \beta & \cos \beta & 0 & a \sin \beta \\ 0 & 0 & 1 & 0 \\ 0 & 0 & 0 & 1\end{array}\right]$.

$\alpha$--The angle from system $O_{3}$ to system $O_{4}$ according to the right-hand rule.

$\beta$--The angle from system $O_{4}$ to system $O_{5}$ according to the right-hand rule.

$a$--The offset distance from system $O_{4}$ to system $O_{5}$. 
Therefore, it can be conclude that

$$
T_{W}={ }_{0}^{1} R_{1}^{2} R_{2}^{3} R_{3}^{4} R_{4}^{5} R=\left[\begin{array}{cccc}
\cos \alpha \cos \beta & -\cos \alpha \sin \beta & \sin \alpha & a \cos \alpha \cos \beta+x \\
\sin \alpha \cos \beta & -\sin \alpha \sin \beta & -\cos \alpha & a \sin \alpha \cos \beta+y \\
\sin \beta & \cos \beta & 0 & a \sin \beta+z \\
0 & 0 & 0 & 1
\end{array}\right] .
$$

Assume the position and pose of the end of robot end is $T_{P}$.

$$
T_{P}=\left[\begin{array}{cccc}
n_{x} & o_{x} & a_{x} & p_{x} \\
n_{y} & o_{y} & a_{y} & p_{y} \\
n_{z} & o_{z} & a_{z} & p_{z} \\
0 & 0 & 0 & 1
\end{array}\right]
$$

Inverse kinematics means to get the moving distance and joint angles according to the known position and pose of the robot in the world coordinates. Therefore, in terms of robot control, the inverse kinematics is the foundation of the robot trajectory control $[6,7]$.

According to Eq. 10, the solution of inverse kinematics is given as shown in Eq. 11.

$T_{W}=T_{p}$.

$\left\{\begin{array}{l}x=p_{x}-a \cdot n_{x} \\ y=p_{y}-a \cdot n_{y} \\ z=p_{z}-a \cdot n_{z} n . \\ \alpha=-\arcsin a_{x} \\ \beta=\arcsin n_{z}\end{array}\right.$

\section{Vision Module}

In the visual servo system based on image feature, the vision module is a key link. The actual precision of the image coordinate will affect the tracking precision of the system.

This module is built based on VS2010 and OpenCV. The main steps are shown in Fig. 5.

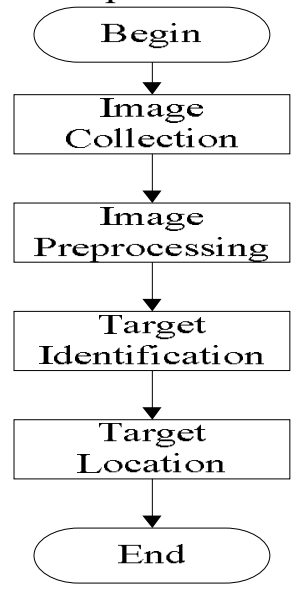

Fig. 5: Flow diagram of image processing

Image Acquisition. In the stage of image collection, a function named cvQueryFrame from the library of OpenCV is circularly used to shoot video. The function is to read next frame all the time, so the essence of video is rapidly playing the frames. If the image read from camera is the first frame, the procedures will apply for memory space and initialize it. Afterwards, images are captured every certain number of frames [8]. As long as the sampling frequency is higher than the observing frequency of eyes, it is enough for playing the video real-time. Setting the sampling interval is $n$, and the whole process is shown in Fig. 6. 


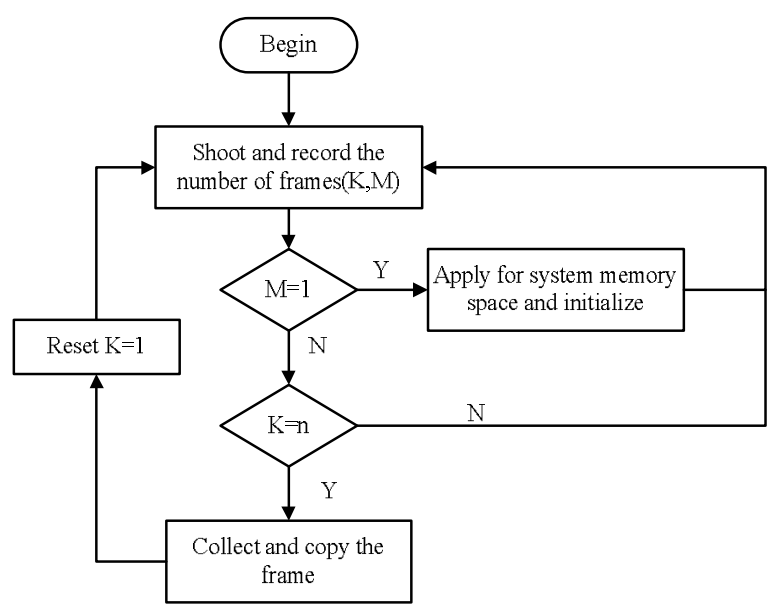

Fig. 6: Flow diagram of image collection

Image Processing. During transmission and storage, information of image feature is inevitably affected by noise in some degree. Therefore, technology of image enhancement is the necessary steps in the vision module. The principle of image enhancement is to highlight the interested feature and weaken the uninterested feature [8]. In the experiment, color images are transformed into gray images, and the image noise is reduced through the medium filtering.

Target Identification. In the paper, the main method is color identification, edge detection and shape recognition.

Target Location. Target localization relies on the binary image generated by image processing. In the binary image, the target is white with black background. By using the method of traversing the pixels of the image, the procedure can calculate center point coordinate of the target. Then the actual distance between the target and camera is obtained by a certain percentage of transformation. After that, upload the coordinates to the controller in order to drive the robot to move to the expected position.

\section{Software Implementation of the System}

VS2010 is used to develop the software system and also used to build the communication between each module in the system. Pewin32 is management software.

PcommServer is used as communication driver between control software and management software. Shear plate is employed as Inter-process communication between control software and vision module. And the Interface of the control software is shown in Fig. 7.

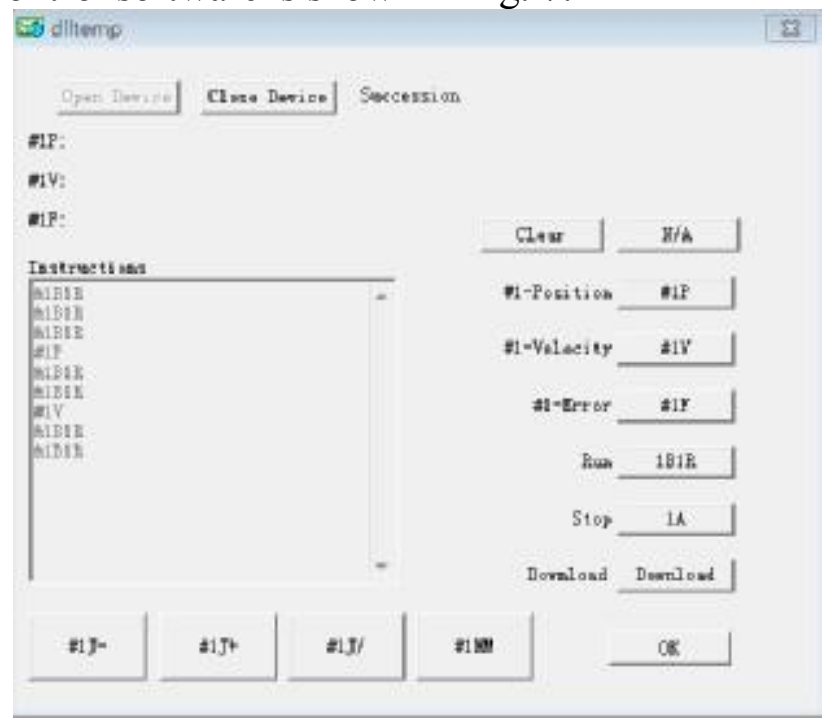

Fig. 7: Interface of the control software 


\section{Experimental Results}

In the experiment, an intelligence car is used as the targrt. Assume that the target moves slowly, then it is enough for the robot to calculation and transmission the data of coordinate without delay.

When the target begins to move, the robot will also move to track it. The tracking error is obtained by the software named PmacPlot32Pro2 as shown in Fig. 8.

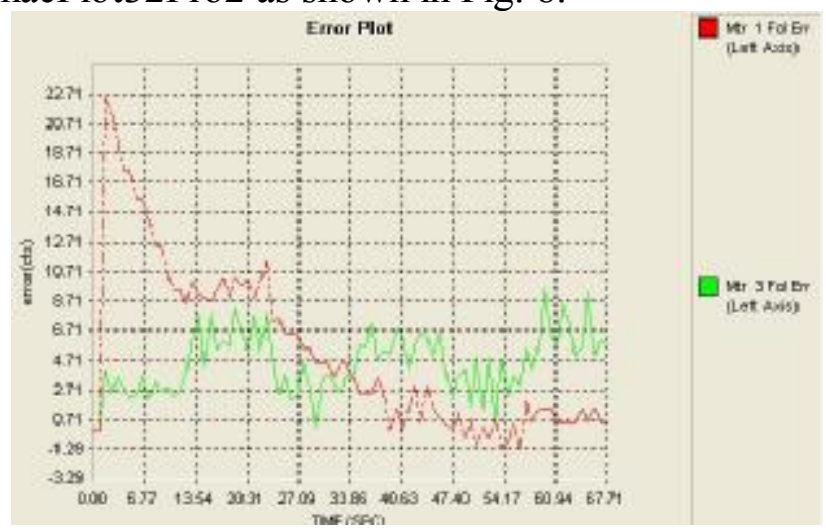

Fig. 8: Plot of tracking error

$\mathrm{X}$-axis: $460000[\mathrm{cts}] \cong 700[\mathrm{~mm}]$.

Y-axis: $478000[\mathrm{cts}] \cong 485[\mathrm{~mm}]$.

From the Fig. 8 and the conversion relationships, it proves that the tracking error is less than 20cts, and it is equivalent that the tracking error is less than $0.1 \mathrm{~mm}$. Obviously, the visual servo robot tracking system is enough effective to track a moving target.

\section{Conclusions}

In the paper, a visual servo robot tracking system is developed completely independently. And the purpose is to design a visual servo robot system to finish some tasks which is impossible for human beings. Experiments show that the visual servo robot can track the target with good performance of real time and tracking precision. The system also has advantages of convenient operation, economic and practical. Therefore, with the visual servo robot system, visual servo robot can replace workers to achieve more difficult tasks.

\section{References}

[1] Piepmeier, J.A, McMurray, G.V, Lipkin, H. Uncalibrated dynamic visual servoing[J]. IEEE Transactions on Robotics and Automation, 2004, 20(1): 143-147.

[2] Elbanhawi, M, Simic, M, Jazar, R. Autonomous Robots Path Planning: An Adaptive Roadmap Approach[J]. ICMRA, 2013, 373-375: 246-254.

[3] Silveira, G, Malis. E. Direct Visual Servoing: Vision-Based Estimation and Control Using Only Nonmetric Information[J]. IEEE Transactions on Robotics, 2012, 28(4): 974-980.

[4] Scheggi, S, Morbidi, F, Prattichizzo, D. Human-Robot Formation Control via Visual and Vibrotactile Haptic Feedback[J]. IEEE Transactions on Haptics, 2014, 7(4): 499-511.

[5] Uke, N.J, Thool, R.C, Moving vehicle detection for measuring traffic count using OpenCV[J]. Journal of Automation and Control Engineering, 2014, 1(4): 349-352.

[6] Pendar, H, Vakil, M, Fotouhi, R, Zohoor, H. Kinematic Analysis of the Spherically Actuated Platform Manipulator[J]. ICRA, 2007, 175-180.

[7] Xuegong D. Research on Robot Control[M]. Hangzhou: Zhejiang University Press, 2007.

[8] Gary, B, Adrian. K. Learning OpenCV[M]. BeijingTsinghua University Press, 2009. 